Research Article

\title{
Dielectric Characteristics of Unsaturated Loess and the Safety Detection of the Road Subgrade Based on GPR
}

\author{
Gao Lv $\mathbb{D}^{1}{ }^{1}$ Jie Yang, ${ }^{1}$ Ning Li, ${ }^{1}$ Dexiu Hu, ${ }^{1}$ Yan Zhang, $^{2}$ and Feng Zhao ${ }^{3}$ \\ ${ }^{1}$ School of Water Resources and Hydro-Electric Engineering, Xi'an University of Technology, Xi'an 710048, China \\ ${ }^{2}$ School of Architecture and Civil Engineering, Xi'an University of Science and Technology, Xi'an 710054, China \\ ${ }^{3}$ Geochemical Exploration Team of Shaanxi Geological Mineral Survey Group, Xingfu Road No. 7, Xincheng District, \\ Xi'an 710043, China \\ Correspondence should be addressed to Gao Lv; lvgao116@xaut.edu.cn
}

Received 28 July 2017; Revised 16 November 2017; Accepted 26 November 2017; Published 31 January 2018

Academic Editor: Lei Yuan

Copyright ( 2018 Gao Lv et al. This is an open access article distributed under the Creative Commons Attribution License, which permits unrestricted use, distribution, and reproduction in any medium, provided the original work is properly cited.

\begin{abstract}
We present a moisture content and permittivity model to simultaneously detect and estimate defects in loess subgrade. Based on the ground-penetrating radar (GPR) method, the dielectric properties of loess in the northwest of China and the imaging feature of the moisture content of different strata were studied. The relative permittivity of loess with different moisture contents was experimented in the laboratory. It was found that the relative permittivity of unsaturated loess was positively related to moisture content. The relationship between relative permittivity and moisture content in different antenna frequencies of GPR was analyzed. Electromagnetic wave reflection rules in the loess interface were studied using the numerical method with different moisture contents. With the increase in moisture content, the amplitude of GPR was increased. When the above conclusions were applied in the engineering practices, there are good effects to detect the defects of the road subgrade. It is a significant guidance for determining the qualitative research of defects in the roadbed.
\end{abstract}

\section{Introduction}

Loess subgrade in the area of northwestern China often appears to collapse, to settle, and to have other problems $[1,2]$.

So it is necessary to earlier detect the defects and cavities in the road subgrade. Then, targeted prevention and treatment will be applied, and the potential losses caused by roadbed subsidence will be avoided. At present, there are many kinds of nondestructive testing (NDT) technologies of subgrade defect, for example, groundpenetrating radar (GPR), hammer sounding, acoustic tomography, falling weight deflectometer (FWD), and time-domain reflectometry (TDR) [3]. Hereinto, GPR is a kind of geophysical detection technology based on electromagnetic wave, which is nondestructive, low-cost, portable, effective, fast, and accurate [4]. The detection principle of GPR is as follows: When the emitted electromagnetic waves (frequency range is $10-2200 \mathrm{MHz}$ ) transmit in the underground, the electromagnetic waves, arriving at the interface of inhomogeneous permittivity, will produce the phenomenon of reflection and refraction. And then, the signals of reflected waves, that is, echo, can reflect the characteristics of underground medium $[5,6]$. As a nondestructive testing technology, GPR has received more and more attention [7-9].

The main wave characteristics of GPR data received by sounding road pavement deformations were revealed by the theoretical and experimental studies of subgrade soils of roads [10]. In order to achieve the best methodology for subgrade cracking detection, different GPR systems, both antenna configuration and frequencies, were tested [11]. Experimental results from field and laboratory investigations using a ground-coupled GPR, dielectric measurement, magnetic imaging tomography (MIT), and DCP tests were presented [12]. Several GPR methods and analysis techniques were used to nondestructively investigate the electromagnetic behavior of subasphalt compacted clayey layers and subgrade soils in unsaturated conditions [13]. 


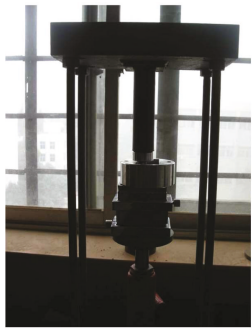

(a)

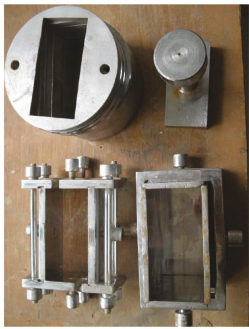

(b)

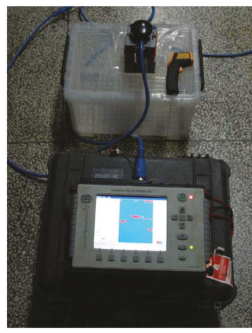

(c)

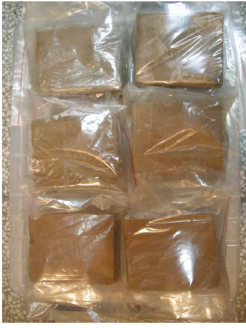

(d)

FIGURE 1: (a) Compacted equipment, (b) sample moulds, (c) GPR device, and (d) the prepared loess samples.

At present, the study of permittivity of unsaturated loess is few, and the direct measurement of the permittivity of unsaturated loess by GPR has little result in this field. In this paper, the empirical relationship between permittivity and unsaturated loess moisture content was obtained by the direct test with the different frequencies of the GPR antenna. The mechanism and law of the GPR wave reflection imaging in the unsaturated loess interface have been studied. The above results were directly applied to detect defects in an engineering project.

\section{Dielectric Property of the Loess}

2.1. Experimental Procedures and Test Parameters. In this paper, loess is the object of the study. The GPR (SIR-3000 series, Geophysical Survey Systems Inc.) was employed to measure the permittivity of loess in laboratory experiment. The experimental procedures are the following:

(1) The soil samples were collected from a construction site and sealed for later use.

(2) The soil samples were manually screened in order to obtain the fine powder.

(3) The samples in which the moisture content was from $10 \%$ to $37 \%$ were prepared.

(4) The plane strain test apparatus was utilized for the sample preparation. The size was $5 \mathrm{~cm}$ by $10 \mathrm{~cm}$ by $10 \mathrm{~cm}$. The reaction frame was utilized for pressuring samples. After 48-hour standing, the reaction frame was used to compact samples. Then, the soil samples were sealed in plastic bags.

(5) According to the sizes of the samples, the selected antenna frequencies were $400 \mathrm{MHz}, 900 \mathrm{MHz}$, and $2 \mathrm{GHz}$, respectively. The soil samples were scanned, and the data was collected.

(6) The measured data postprocessing was done by the RADAN5 Software.

The devices in the experiment and the prepared soil samples are shown in Figure 1, and the antenna parameters of GPR are shown in Table 1.

2.2. Test Principle of the Loess Permittivity. The calculation model is shown in Figure 2. The travel time of the GPR wave
TABLE 1: The antenna parameters of GPR.

\begin{tabular}{lccc}
\hline Frequency & $2 \mathrm{GHz}$ & $900 \mathrm{MHz}$ & $400 \mathrm{MHz}$ \\
Emissivity & $350 \mathrm{kHz}$ & $100 \mathrm{kHz}$ & $100 \mathrm{kHz}$ \\
Ranges (ns) & 12 & 20 & 40 \\
Scanning speed (scan/s) & 300 & 50 & 50 \\
Gain & 1 & 2 & 5 \\
\hline
\end{tabular}

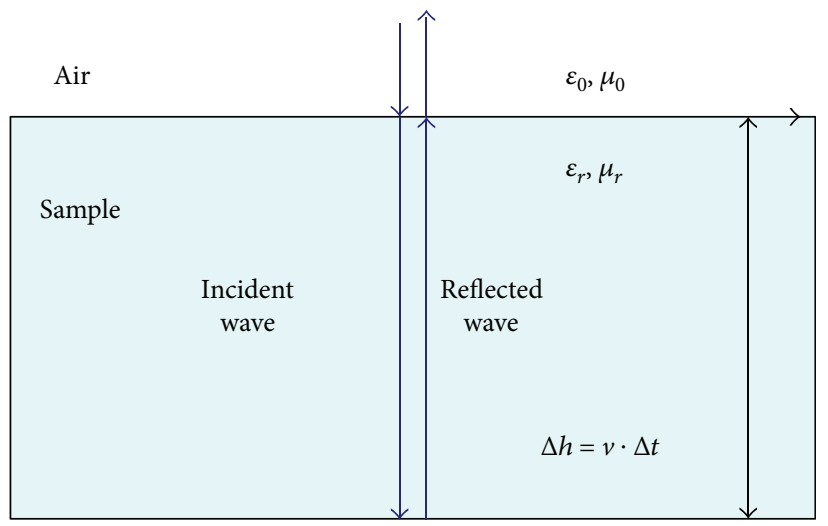

Figure 2: Wave velocity calculation model of GPR.

in the medium is also the object of the study. The reflection positions of electromagnetic wave in the sample interfaces are analyzed. Meanwhile, according to the travel time, the actual wave velocity of the GPR wave can be calculated.

The electromagnetic waves propagate in the loess samples and reach the bottom of the sample after the travel time $\Delta t$. Then, the reflected part of the electromagnetic waves will propagate to the surface of the loess samples after the travel time $\Delta t$ and will be received by GPR equipment. The value of the travel time $\Delta t$ can be read from the scanning data of the GPR. Meanwhile, the travel length of the electromagnetic wave $\Delta h$ can be directly measured by the size of the sample. Therefore, the actual wave velocity of the electromagnetic waves propagating in the loess samples can be calculated by the formula $v=\Delta h / \Delta t$. The relative permittivity of the loess samples can be calculated as follows:

$$
\sqrt{\varepsilon_{r}}=c \frac{\Delta h}{\Delta t} .
$$

2.3. Calculation of the Permittivity. The soil samples were scanned by the GPR antenna. The antenna frequencies were 


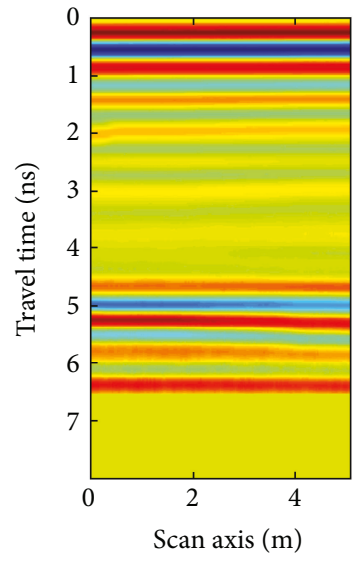

(a)

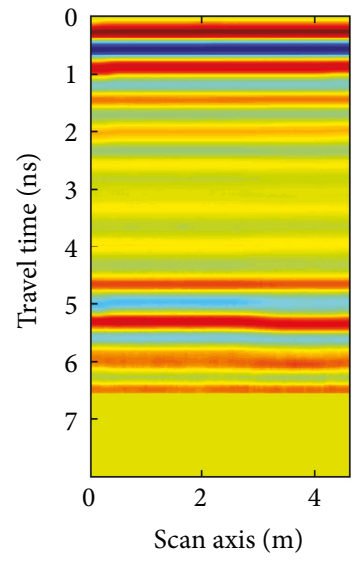

(b)

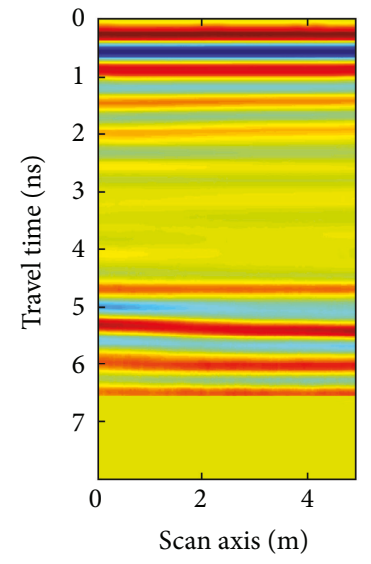

(c)

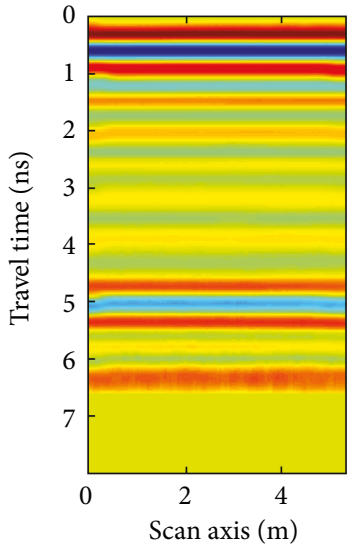

(d)

Figure 3: (a-d) The GPR images of loess with the moisture contents 10\%, 19\%, 28\%, and 37\%, respectively.

TABLE 2: Loess permittivity with different moisture contents under different frequencies.

\begin{tabular}{lccc}
\hline Moisture content $(\%)$ & $\varepsilon_{\text {RADAN900 }}$ & $\varepsilon_{\text {RADAN400 }}$ & $\varepsilon_{\text {RADAN2G }}$ \\
\hline 10 & 4.06 & 3.96 & 5.21 \\
13 & 4.82 & 4.26 & 6.01 \\
16 & 5.98 & 5.78 & 7.12 \\
19 & 6.99 & 6.65 & 8.93 \\
22 & 8.98 & 8.37 & 10.98 \\
25 & 11.43 & 10.86 & 12.88 \\
28 & 13.21 & 12.56 & 14.98 \\
31 & 15.43 & 14.77 & 17.12 \\
34 & 18.12 & 17.36 & 19.57 \\
37 & 20.98 & 20.32 & 22.23 \\
\hline
\end{tabular}

$400 \mathrm{MHz}, 900 \mathrm{MHz}$, and $2 \mathrm{GHz}$, respectively. The measured data was processed by the RADAN5 Software through filtering the wave and removing the background noise. The data of $2 \mathrm{GHz}$ as an example are shown in Figure 3.

The different imaging features in the process of the electromagnetic wave propagation are shown in Figures 3(a)$3(\mathrm{~d})$. The reflected waves from $1 \mathrm{~ns}$ to $3 \mathrm{~ns}$ are obvious, due to the surface reflection of loess samples and selfdeconvolution of GPR wavelets. The propagation range of the electromagnetic waves in the samples is mainly from $3 \mathrm{~ns}$ to $6 \mathrm{~ns}$. In this range, there is no obvious reflection of electromagnetic wave. The electromagnetic waves keep good damping characteristics. It is indicated that the samples are relatively homogeneous. The reflected waves from $6 \mathrm{~ns}$ to $8 \mathrm{~ns}$ are due to the reflection of the bottom surface of the samples. Because the change of soil moisture content directly affects the soil permittivity, there must be differences in the GPR images. Therefore, GPR reflection amplitudes increase with the increase in moisture content of soil samples from $10 \%$ to $37 \%$.

The permittivity of the samples with different moisture contents via applying different frequencies can be calculated by (1). The values of the permittivity are shown in Table 2.
For the same sample, different frequencies can induce the different values of permittivity. The reason is that the electromagnetic waves of different frequencies have differently impacted the intrinsic nature of loess permittivity. Therefore, the same sample exhibits different test values of the permittivity under different frequencies, which is considered a standard and an important basis of the influence on antenna frequency.

\section{The Relationship of Permittivity and Moisture Content under Different Frequencies}

3.1. Experience Curves of Permittivity and Moisture Content. (1) Topp formula [14], obtained by experiment fitting the empirical cubic formula

$$
\begin{aligned}
\varepsilon_{r}= & 3.03+9.3 \theta_{v}+146.0 \theta_{v}^{2}-76.6 \theta_{v}^{3}, \\
\theta_{v}= & -5.3 \times 10^{-2}+2.92 \times 10^{-2} \varepsilon_{r}-5.5 \\
& \times 10^{-4} \varepsilon_{r}^{2}+4.3 \times 10^{-6} \varepsilon_{r}^{3},
\end{aligned}
$$

where $\varepsilon_{r}$ is the permittivity and $\theta_{v}$ is the volume of moisture content. In (2), the moisture content is used to calculate the permittivity. On the contrary, the permittivity is used to calculate the moisture content in (3).

(2) CRIM (complex reflective index model) [15] formula, an arithmetic square root model

$$
\sqrt{\varepsilon}=(1-\phi) \sqrt{\varepsilon_{s}}+S_{w} \phi \sqrt{\varepsilon_{w}}+\left(1-S_{w}\right) \phi \sqrt{\varepsilon_{a}} .
$$

The subscripts $s, w$, and $a$ represent the soil, water, and air, respectively. $\varphi$ represents the moisture content, and $S_{w}$ is on behalf of the moisture saturation. On the condition that the water permittivity is 81 and the loess permittivity is 5 , so (4) can be simplified as

$$
\sqrt{\varepsilon}=\phi \sqrt{\varepsilon_{m}}+(1-\phi) \sqrt{\varepsilon_{w}} .
$$

(3) Dobson [16] formula

$$
\varepsilon_{\text {soil }}^{\alpha}=1+\frac{\rho_{b}}{\rho_{\mathrm{ss}}}\left(1-\varepsilon_{\mathrm{ss}}^{\alpha}\right)+m_{v}^{\beta}\left(\varepsilon_{f w}^{\alpha}-1\right) .
$$


$\varepsilon_{\text {soil }}$ refers to the soil permittivity. $\rho_{b}$ and $\rho_{\text {ss }}$ refer to the densities of dry soil and soil skeleton, respectively. $E_{\mathrm{ss}}$ refers to the solid permittivity. $m_{v}$ refers to the solid quality. Empirical coefficient $\alpha$ is 0.65 and $\beta$ is $1.0 \sim 1.17$ (from sandy soil to clay).

3.2. The Measured Permittivity Results under Different Frequencies. The samples with different moisture contents were tested by the antenna of $400 \mathrm{MHz}, 900 \mathrm{MHz}$, and $2 \mathrm{GHz}$. The scatter diagrams of the permittivity and moisture content were fitted. The optimum fitting curves are shown in Figure 4.

As shown in Figure 4, the experimental results are near to the experiential fitting curves. It demonstrates the correctness of the experimental results. Under the same frequency of the GPR antenna, sample permittivity is increased with the increase in moisture content. At the same time, the permittivity of the sample with the same moisture content is also increased with the increase in frequency. The antenna frequencies applied in the experiment are $400 \mathrm{MHz}, 900 \mathrm{MHz}$, and $2 \mathrm{GHz}$, respectively. So the permittivity of the antenna frequency of $400 \mathrm{MHz}$ is minimal, and that of $2 \mathrm{GHz}$ is maximal.

There is a distance between the blue points and the formula lines (black). It could be briefly analyzed as the following. First, the GPR method is a nondestructive method. So it is always less accurate than an empirical formula established by an invasive method. Next, the GPR method is easily subject to external disturbances.

The conjugate gradient method is used to describe the mathematical relationship between the moisture content and permittivity. Meanwhile, the linear models are established according to the former testing data.

$$
\begin{aligned}
& \varepsilon_{r}=0.61 \cdot \theta_{v}+1.58, f=400 \mathrm{MHz}, \\
& \varepsilon_{r}=0.63 \cdot \theta_{v}+1.81, f=900 \mathrm{MHz}, \\
& \varepsilon_{r}=0.64 \cdot \theta_{v}+3.18, f=2 \mathrm{GHz} .
\end{aligned}
$$

The fitting calculation results show that the conjugate gradient method can solve the correction factors well in one dimensional model. And the model can be applied directly to the practice and is a reliable tool for moisture content of similar projects.

\section{Numerical Simulation of the Interface Reflection Mechanism of Unsaturated Loess}

Compared with the experimental method, an ideal effect of wave propagation can be received by the numerical simulation method. The numerical method can avoid the defects of the test method, such as inhomogeneous medium, uneven compaction degree of large-scale samples, and measurement error. In this section, imaging features of different permittivity values were comparatively analyzed. Further, the influence of the dielectric constant on radar echo waveform was studied.

4.1. Geoelectric Model of Interface Reflection of Unsaturated Loess. GprMax2.0 software is used for numerical simulation

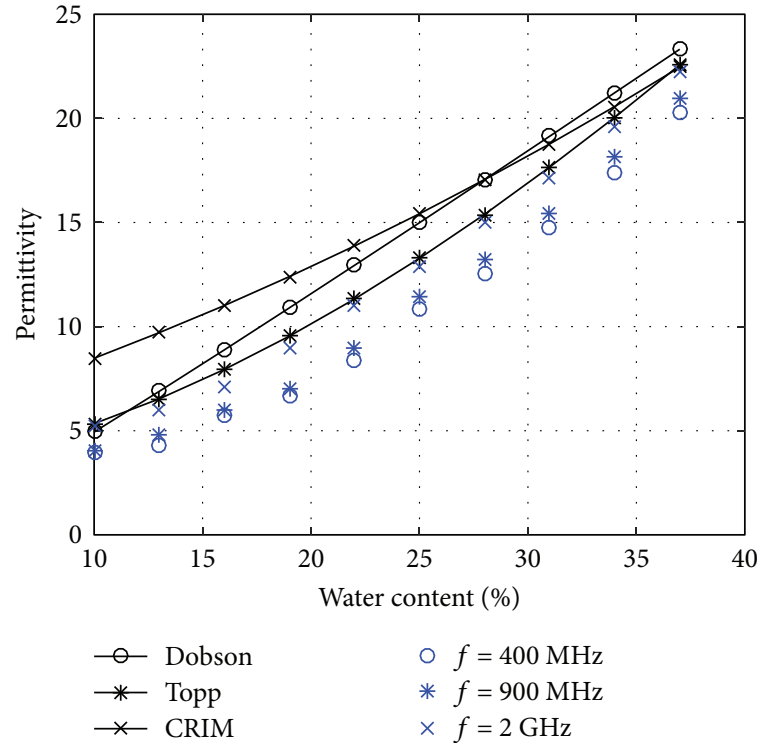

FIgURE 4: The experiential fitting curves and experimental results of the loess with different moisture contents under different frequencies (the black symbols represent the experiential results, and the blue symbols represent the experimental results).

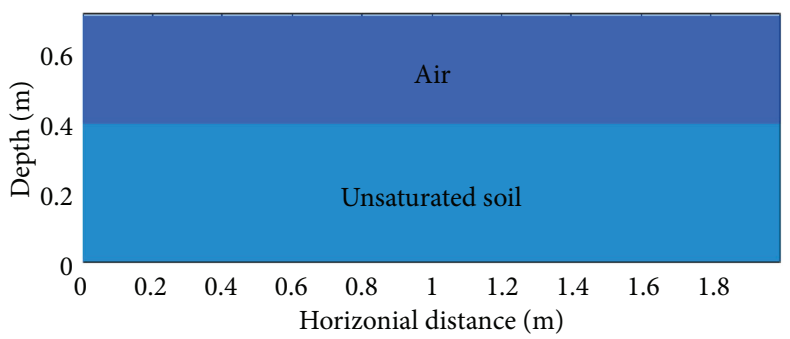

Figure 5: The schematic diagram of the numerical model of unsaturated loess.

in this article. GprMax is open-source software that simulates electromagnetic wave propagation. It solves Maxwell's equations in 3D using the finite-difference time-domain (FDTD) method. GprMax was designed for modelling GPR [17-19].

The geoelectric model of shallow loess was designed according to the waveform comparison. Keeping the other parameters held, the influence of permittivity on GPR imaging was investigated and the quantification of the echo waveform was deeply analyzed. The schematic diagram of the specific geoelectric model is shown in Figure 5.

The horizontal distance of this model is $2.0 \mathrm{~m}$. The depth is $0.7 \mathrm{~m}$. The cell size is $0.0025 \mathrm{~m}$ by $0.0025 \mathrm{~m}$. The time depth is $20 \mathrm{~ns}$. The parameters of the first layer are as follows. The permittivity is 0 . The electrical conductivity is $0.00 \mathrm{~S} / \mathrm{m}$. The thickness of the first layer is $30 \mathrm{~cm}$. The permittivity values of the second layers are 5, 10,20,30,40, and 50, respectively. The electrical conductivity is $0.00001 \mathrm{~S} / \mathrm{m}$. The thickness of the second layer is $0.4 \mathrm{~m}$. The wavelet frequency is set as $900 \mathrm{MHz}$. The excitation source is the Ricker wavelet. In the numerical simulation, there are 180 step calculations and each step calculation contains 3391 times. 


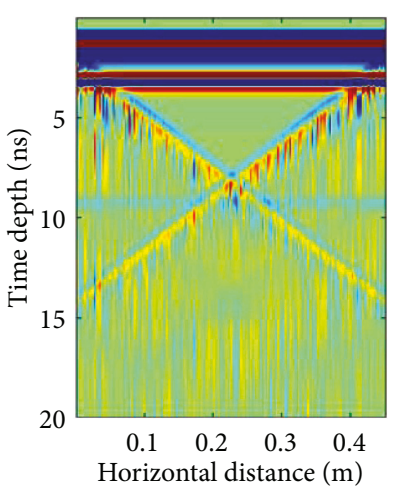

(a)

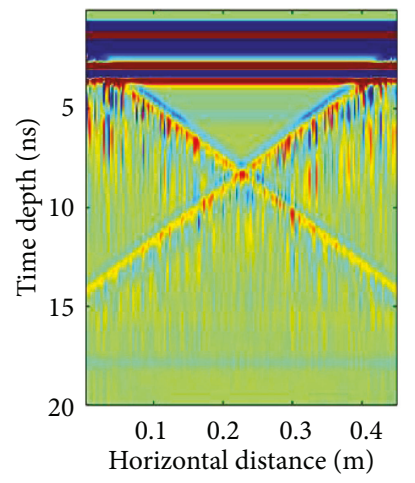

(d)

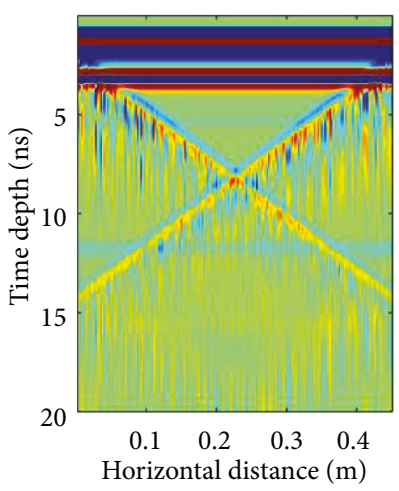

(b)

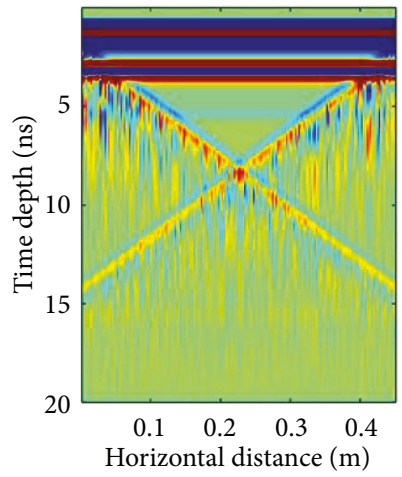

(e)

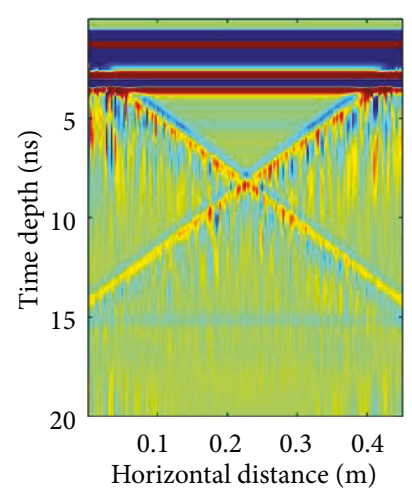

(c)

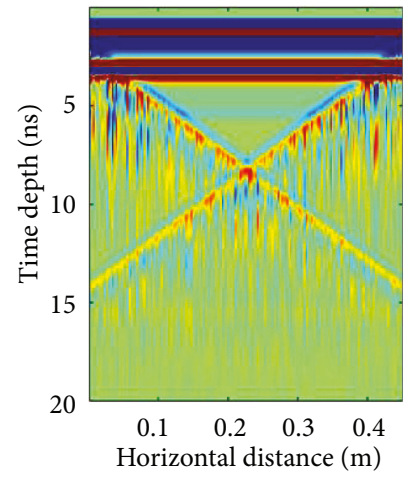

(f)

FIGURE 6: (a-f) The GPR imaging of the subgrade of unsaturated loess when $\varepsilon_{r}$ are $5,10,20,30,40$, and 50 , respectively.

\subsection{The Amplitude Rule of Reflected Wave on Different} Permittivity Layers. According to the above numerical calculation, the reflected characteristics of the GPR waves in different moisture content interfaces can be observed. The permittivity values of the grassroot level of unsaturated loess were set as $5,10,20,30,40$, and 50 , respectively. Then, the simulate calculation was done. The stratigraphic sections were obtained and shown in Figure 6.

In Figure 6, the interface reflection trend of permittivity is obvious. The reflection amplitude shows an increasing trend at the position of $3 \mathrm{~ns}$. It is indicated that the interface inflection obviously increases with the increase in the moisture content. But it cannot give quantitative descriptions only from the stratigraphic sections. So it is necessary to comparatively analyze the amplitude value.

The method extracts the single wave in the forward modeling data. Obviously, the interface reflection interval is presented as the characteristics of "positive-negativepositive." This dissertation applies the data of peaks and troughs, as shown in Figure 7.

In Figure 7, the first crest of the echo curves of interface reflection appears when STEPS $=490$. The wave trough appears when STEPS $=545$, and the absolute value of the wave valley is the maximum point of the reflection amplitude. With the increases in moisture content on the interface, the reflection amplitudes of GPR are enlarged accordingly. There is a clear mathematical relationship between the maximum reflection amplitude and permittivity of medium.

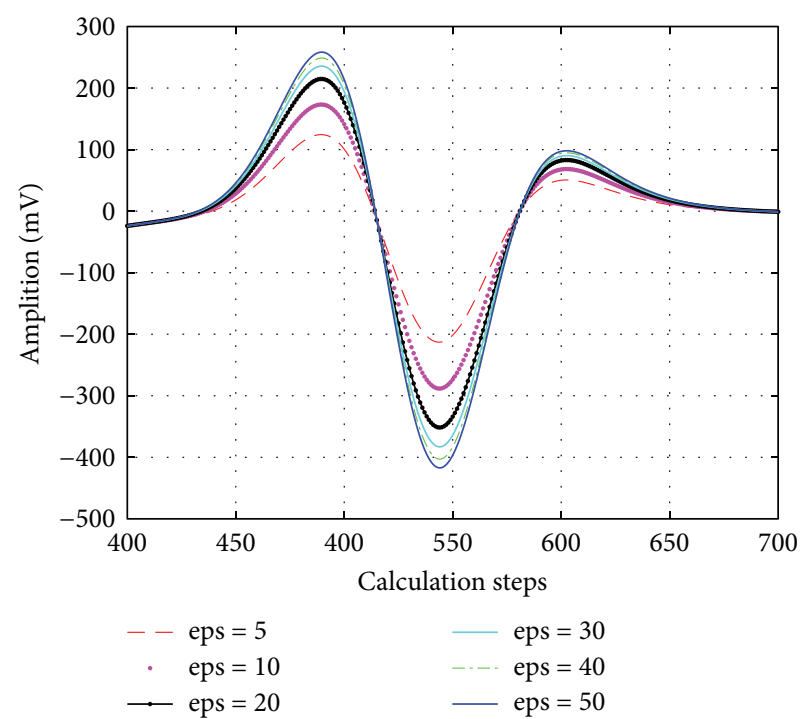

FIGURE 7: Single waves of unsaturated loess interface echoes.

The amplitude values of interface reflection are shown in Table 3.

From the wave amplitudes in Table 3 , it can be clearly seen that the absolute values of the maximum amplitude and minimum amplitude are increased, respectively, with the increase in the permittivity of the loess. 
TABLE 3: Reflection amplitude under different moisture contents on the loess interface.

\begin{tabular}{lcccccc}
\hline & $\varepsilon_{r}=5$ & $\varepsilon_{r}=10$ & $\varepsilon_{r}=20$ & $\varepsilon_{r}=30$ & $\varepsilon_{r}=40$ & $\varepsilon_{r}=50$ \\
\hline Amplitude maximum & 124.37 & 173.19 & 214.77 & 235.61 & 248.89 & 258.37 \\
Amplitude minimum & -212.89 & -288.34 & -351.61 & -383.03 & -402.97 & -417.21 \\
\hline
\end{tabular}

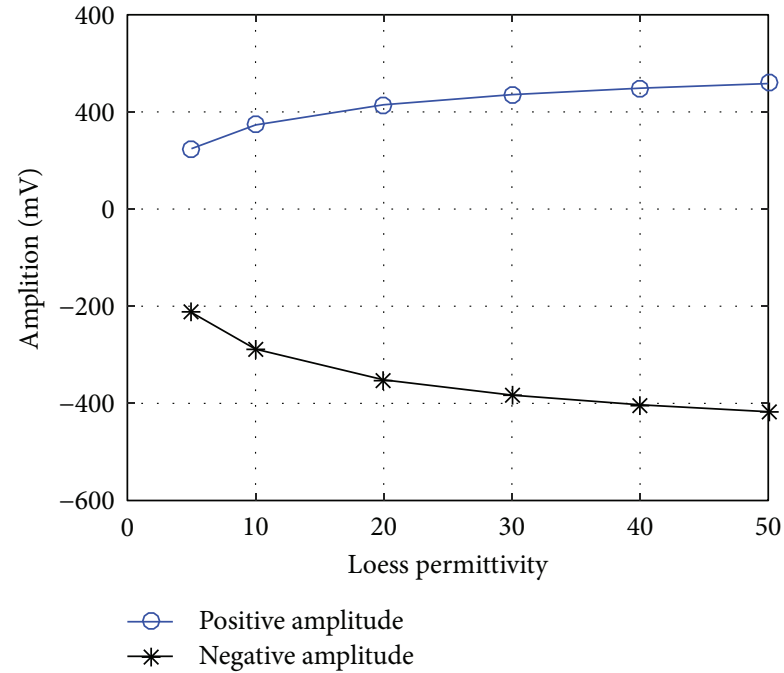

Figure 8: Amplitudes of unsaturated loess interface echoes.

Therefore, the mathematical relation between the reflection amplitude of loess interfaces and permittivity is obtained and shown as follows.

$$
\begin{aligned}
\text { Peak: } A_{\text {peak }} & =-0.08 \varepsilon_{r}^{2}-7.04 \varepsilon_{r}+100.42, \\
\text { Trough: } A_{\text {trough }} & =0.12 \varepsilon_{r}^{2}-10.79 \varepsilon_{r}-176.5
\end{aligned}
$$

Based on the result of numerical calculation, the empirical correlation of the reflection amplitude and permittivity is shown in Figure 8.

\section{Actual Measurements and Analysis of Loess Roadbed Diseases}

5.1. Project Overview. The Bin He Road built in 2013 is isolated on the south of Wei He River in Baoji city, China. The road was established on the site of the river bed post, and the foundation is roller-compacted loess. In 2016, sewage pipes were laid at the depth of 10 meters below the surface using the straight push method. And then, poor geological disasters arise, such as surface collapse and surface subsidence. The specific positions are pinpointed in Figure 9.

5.2. The GPR Sections of the Subgrade before and after Road Maintenance. According to the practical situation in the spot, the pavements above the sewage pipe were scanned directly using the $200 \mathrm{MHz}$ and $400 \mathrm{MHz}$ antenna, respectively. The testing purpose is to identify the subgrade of the abnormal area which is located between the road surfaces and pipes. Furthermore, the hidden area of risk will be repaired early, and the sinking or void of the road will be avoided.
The geological sections of $40 \mathrm{~m}$ length at the horizontal distance between point 360 and point 380 of the Bin He Road were extracted. The GPR grams of the subgrade before and after road maintenance are shown in Figures 10 and 11 , respectively.

These comparison sections show the profile of before and after maintenance using the GPR antenna of $200 \mathrm{MHz}$. Figure 10(a) shows the obvious points, such as D1, D2, D3, D4, D5, and D6. Hereinto, D2 and D5 are near-surface reflection anomalies. Both of them belong to very serious potential hazards. The reflection signal intensity curves of D1 were inferred as water-rich drawbacks (refer to the laws of Section 4.2).

To avert disasters, the test results were immediately sent to the administration. The measured locations were repaired. And then, the former areas were measured again. The geological sections are shown in Figure 10(b). The consolidated methods were timely taken, and the dangerous situation of D2 and D5 disappeared. In the meantime, the foundation of D1 and D4 was also consolidated and compacted.

These comparison sections show the profile of before and after maintenance using the GPR antenna of $400 \mathrm{MHz}$. Figure 11(a) shows the obvious points such as $d 1, d 2$, and d3. Hereinto, d2 and d3 are near-surface reflection anomalies corresponding to the D2 and D5 in Figure 10(a). In the similar way, d1 corresponds to D1. Apparently, there are certain differences in estimating depth and radius of the targets by different frequency antennas.

According to the specific points to find out the defect and repaired, there is no remarkable abnormality as shown in Figure 11(b). Timely detection and maintenance of the roadbed are effective ways to enhance the integrity and stability of the subgrade. The hidden trouble of the structure can be detected in time, and accident can be avoided by the damage detection and diagnosis.

The antenna of GPR has a constraint condition they must simultaneously satisfy: detecting depth and detecting precision. In general, the bigger the value of the principal frequency, the smaller the detecting depth. By comparing the quantity of the target exposed in Figures 10 and 11, it can be seen that the measurements using the $200 \mathrm{MHz}$ GPR antenna are more accurate. And the antenna frequency change plays a vital role in the precision, which reflected the general rule.

The field excavation of the D1 point is shown in Figure 12.

The point D1 excavation verifies that the anomaly characteristics of the GPR imaging accord with the true position of the subterranean cavitation. The horizontal position about 360 meters has a cylindrical hole about $1 \mathrm{~m}$ in diameter and $1 \mathrm{~m}$ deep. This shows that geophysical methods have prompt, high-precision, and nondevastating advantages in environmental protection. 


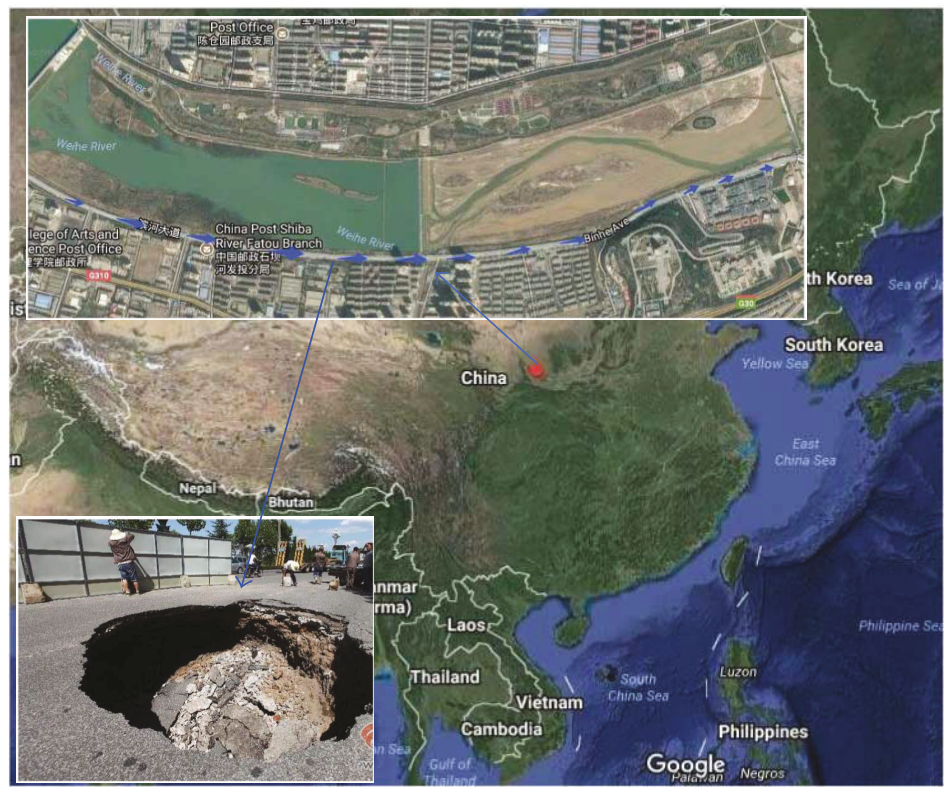

Figure 9: Location of the project.

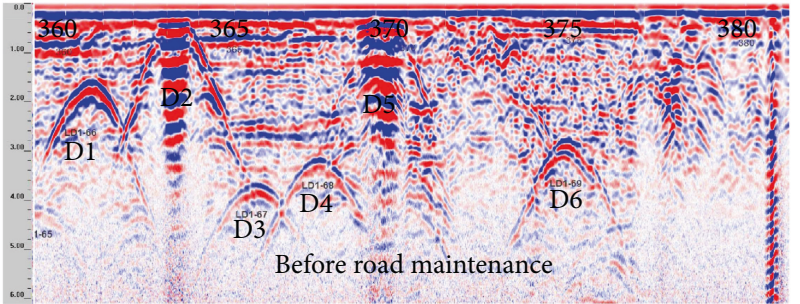

(a)

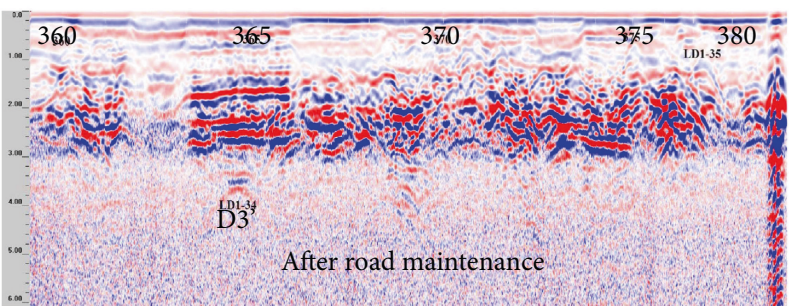

(b)

Figure 10: (a) GPR sections by the $200 \mathrm{MHz}$ antenna before road maintenance. (b) GPR sections by the $200 \mathrm{MHz}$ antenna after road maintenance.

\section{Conclusions}

Through the research on the loess permittivity at different moisture contents and the influence of the antenna frequency on the loess permittivity, the conclusions are as follows:

(1) The experiment and comparison results show that the loess permittivity increases along with the broadening of moisture content in the loess soil. In addition, the same sample produces different permittivity values under different frequencies, and the

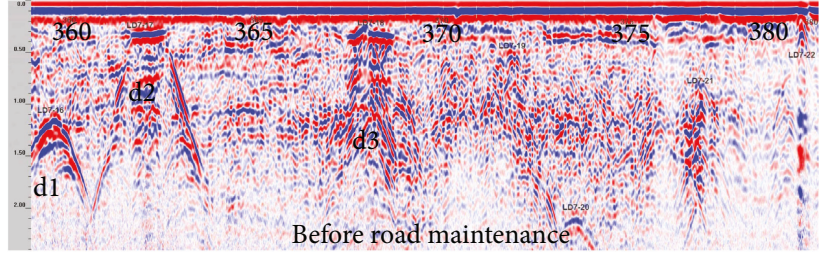

(a)



(b)

FIgURE 11: (a) GPR sections by the $400 \mathrm{MHz}$ antenna before road maintenance. (b) GPR sections by the $400 \mathrm{MHz}$ antenna before road maintenance.

loess permittivity increases when the frequencies are increased.

(2) With the increase in the moisture content in unsaturated loess, the permittivity and the interface reflection amplitudes markedly improved. There is a corresponding increase in the absolute value of the maximum amplitude and the minimum amplitude of the reflection amplitudes with increasing permittivity. And then, this law is established.

(3) From the study of characteristics of the loess permittivity and the interfacial reflection, we can gain some understanding of actual detection. Systemic discusses with the geology flaw recognition and flaw measurements have been made. 


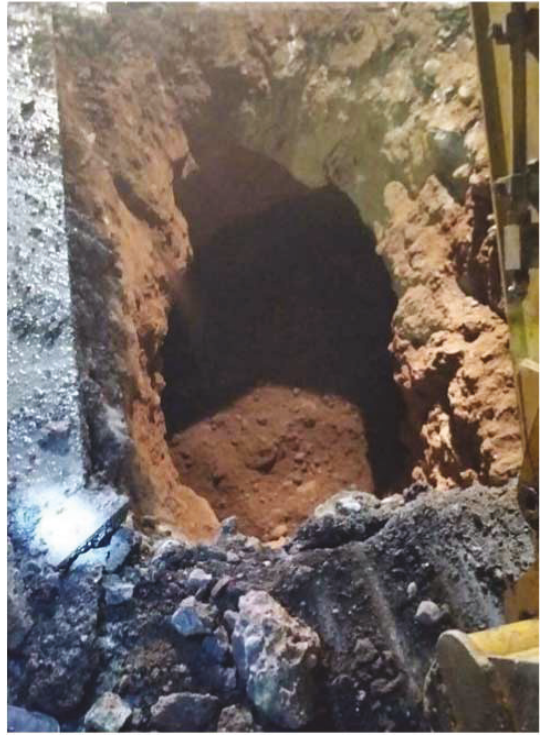

Figure 12: The verification of excavation.

\section{Conflicts of Interest}

The authors declare that they have no conflicts of interest.

\section{Acknowledgments}

The authors gratefully acknowledge the financial supports by the China Postdoctoral Science Foundation (Grant no. 2017M613175). And the work was supported by the National Science Foundation of China (Grant nos. 41301597, 11572246, and 51509200) and the Open Foundation of State Key Laboratory Base of Eco-Hydraulic Engineering in Arid Area (Grant no. 2016ZZKT-8).

\section{References}

[1] E. Forte and M. Pipan, "Review of multi-offset GPR applications: data acquisition, processing and analysis," Signal Processing, vol. 132, pp. 210-220, 2017.

[2] P. Dobriyal, A. Qureshi, R. Badola, and S. A. Hussain, "A review of the methods available for estimating soil moisture and its implications for water resource management," Journal of Hydrology, vol. 458-459, pp. 110-117, 2012.

[3] S. K. Verma, S. S. Bhadauria, and S. Akhtar, "Review of nondestructive testing methods for condition monitoring of concrete structures," Journal of Construction Engineering, vol. 2013, Article ID 834572, 11 pages, 2013.

[4] A. Sadoun, M. Broutin, and J. M. Simonin, "Assessment of HWD ability to detect debonding of pavement layer interfaces," in 8th International Conference on Mechanisms of Cracking and Debonding in Pavements, pp. 763-769, Springer, Dordrecht, 2016.

[5] F. Tosti and A. Umiliaco, "FDTD simulation of the GPR signal for preventing the risk of accidents due to pavement damages," International Journal of Interdisciplinary Telecommunications and Networking, vol. 6, no. 1, pp. 1-9, 2014.

[6] T. Inazaki, S. Imamura, H. Kisanuki, and K. Hayashi, "Combined GPR and non-contact surface wave measurements for the investigation of pavement structure," in Symposium on the application of geophysics to engineering and environmental problems, pp. 308-313, Society of Exploration Geophysicists and Environment and Engineering Geophysical Society, Austin, TX, USA, 2015.

[7] K. T. Rudahl and S. E. Goldin, "A Smart Geofabric for Detecting Transportation Substructure Damage," in DEStech Transactions on Engineering and Technology Research, 1st International Conference on Transportation Infrastructure and Materials (ICTIM 2016), pp. 767-773, University of Chang'an Press, Xi'an, 2016.

[8] T. Saarenketo, Electrical Properties of Road Materials and Subgrade Soils and the Use of Ground Penetrating Radar in Traffic Infrastructure Surveys, Oulu university press, Finland, 2006.

[9] A. Benedetto, "Water content evaluation in unsaturated soil using GPR signal analysis in the frequency domain," Journal of Applied Geophysics, vol. 71, no. 1, pp. 26-35, 2010.

[10] R. E. Smith, D. T. Anderson, J. E. Ball, A. Zare, and B. Alvey, "Aggregation of Choquet integrals in GPR and EMI for handheld platform-based explosive hazard detection," in Proceeding Volume 10182, Detection and sensing of mines, explosive objects, and obscured targets XXII, p. 1018217, Anaheim, CA, USA, 2017.

[11] J. Xiao and L. Liu, "Permafrost subgrade condition assessment using extrapolation by deterministic deconvolution on multifrequency GPR data acquired along the Qinghai-Tibet Railway," IEEE Journal of Selected Topics in Applied Earth Observations and Remote Sensing, vol. 9, no. 1, pp. 83-90, 2016.

[12] L. L. Fedorova, D. V. Savvin, M. P. Fedorov, and A. S. Struchkov, "GPR monitoring of cryogenic processes in subgrade soils," in 2016 16th International Conference on Ground penetrating radar (GPR), Hong Kong, 2016, IEEE.

[13] V. Marecos, M. Solla, S. Fontul, and V. Antunes, “Assessing the pavement subgrade by combining different nondestructive methods," Construction and Building Materials, vol. 135, pp. 76-85, 2017.

[14] G. C. Topp, J. L. Davis, and A. P. Annan, "Electromagnetic determination of soil water content: measurements in coaxial transmission lines," Water Resources Research., vol. 16, no. 3, pp. 574-582, 1980.

[15] S. Laurens, J. P. Balayssac, J. Rhazi, G. Klysz, and G. Arliguie, "Non-destructive evaluation of concrete moisture by GPR: experimental study and direct modeling," Materials and Structures, vol. 38, no. 9, pp. 827-832, 2005.

[16] M. C. Dobson, F. T. Ulaby, M. T. Hallikainen, and M. A. El-Rayes, "Microwave dielectric behaviour of wet soil part II: dielectric mixing models," IEEE Transactions on Geoscience and Remote Sensing, vol. GE-23, no. 1, pp. 35-46, 1985.

[17] C. Warren, A. Giannopoulos, and I. Giannakis, "gprMax: open source software to simulate electromagnetic wave propagation for ground penetrating radar," Computer Physics Communications, vol. 209, pp. 163-170, 2016.

[18] I. Giannakis, A. Giannopoulos, and C. Warren, "A realistic FDTD numerical modeling framework of ground penetrating radar for landmine detection," IEEE Journal of Selected Topics in Applied Earth Observations and Remote Sensing, vol. 9, no. 1, pp. 37-51, 2016.

[19] I. Giannakis and A. Giannopoulos, "A novel piecewise linear recursive convolution approach for dispersive media using the finite-difference time-domain method," IEEE Transactions on Antennas and Propagation, vol. 62, no. 5, pp. 2669-2678, 2014. 


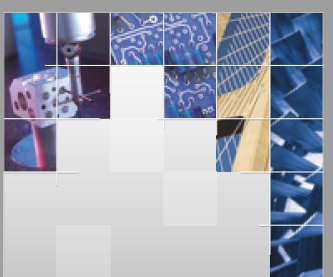

\section{Enfincering}
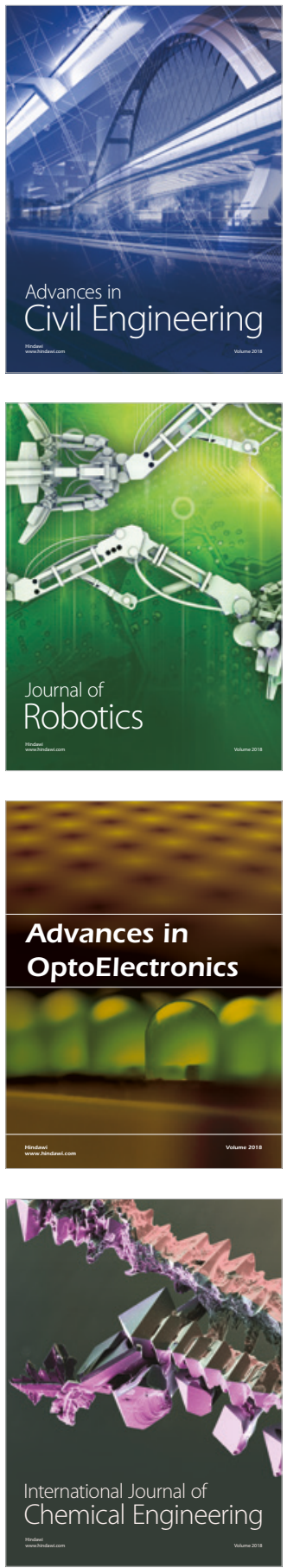

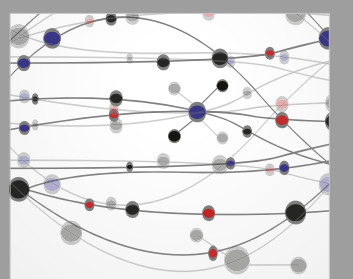

\section{Rotating \\ Machinery}

The Scientific World Journal

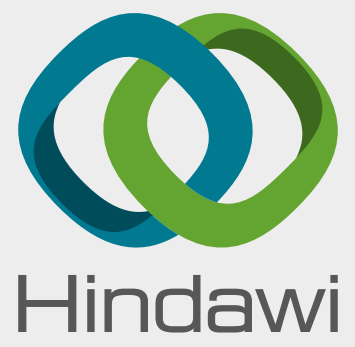

Submit your manuscripts at

www.hindawi.com
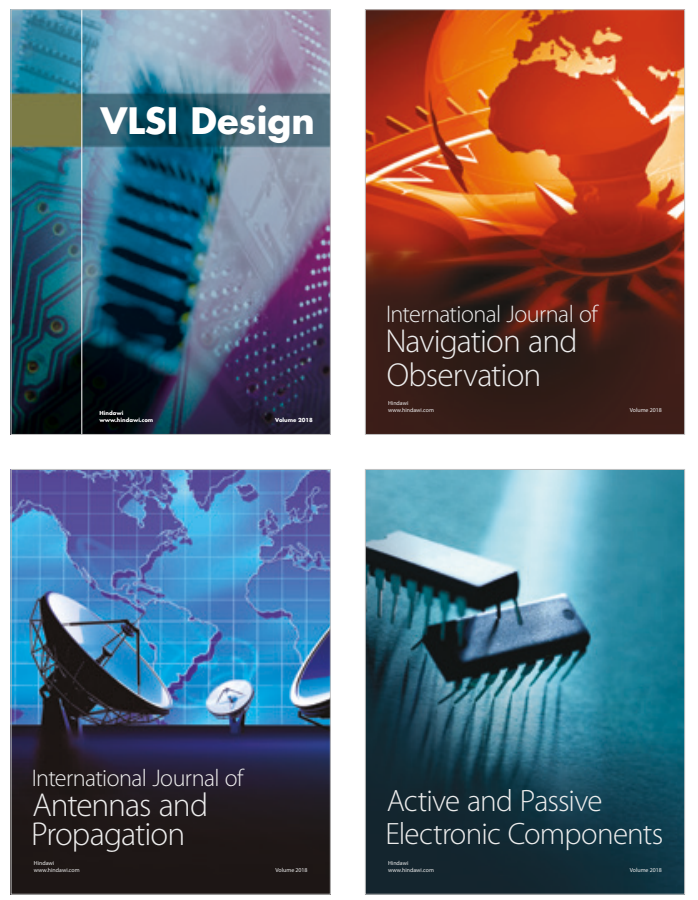
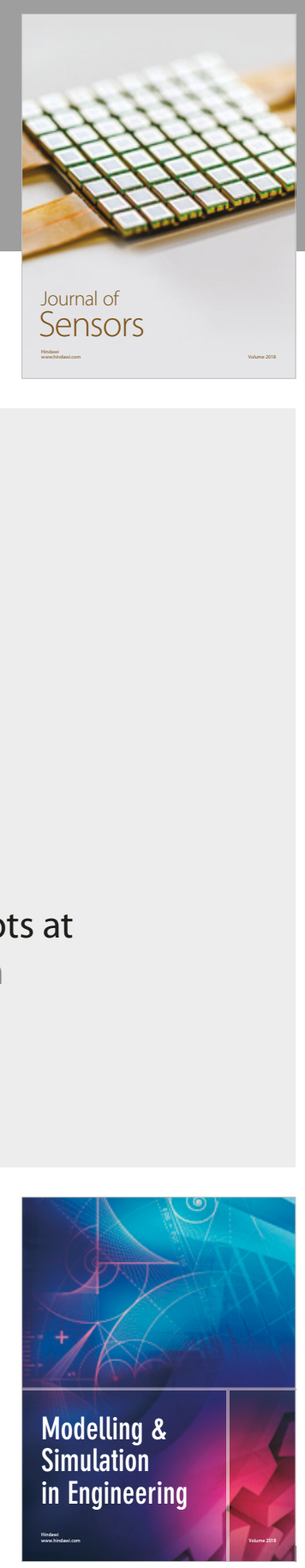

\section{Advances \\ Multimedia}
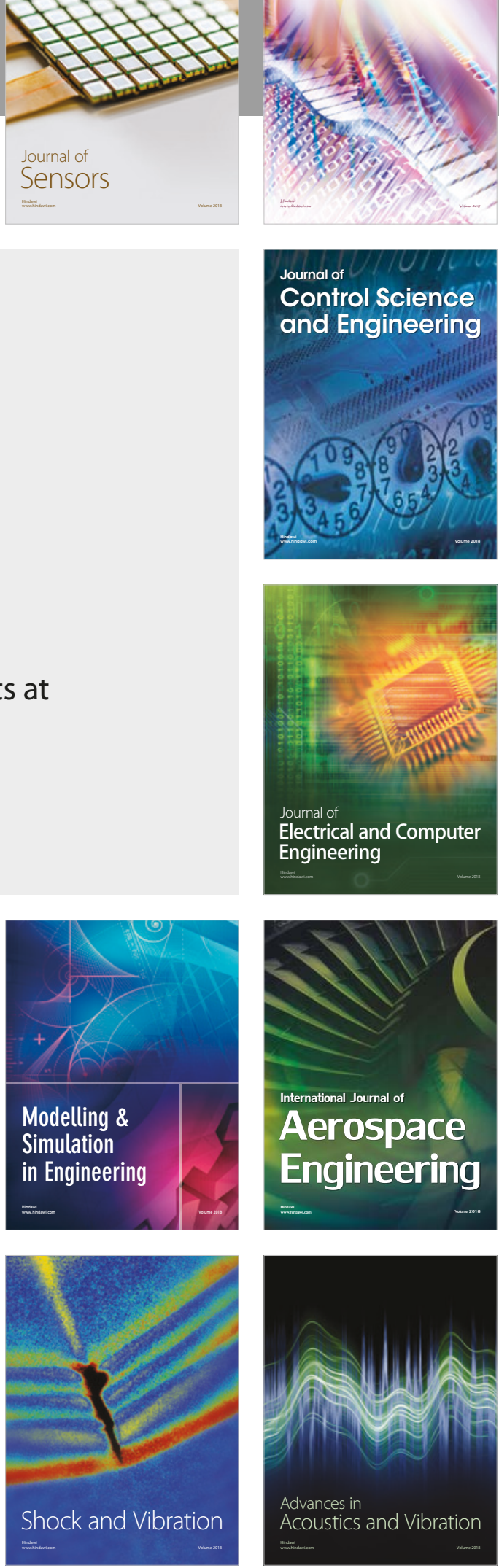